DEPARTMENT OF THE INTERIOR

U. S. GEOLOGICAL SURVEY

\title{
Chemical Analyses of Volcanic Rocks from Monogenetic and Shield Volcanoes near Crater Lake, Oregon
}

by

\author{
Peggy E. Bruggman 1 \\ Charles R. Bacon ${ }^{1}$ \\ J. Steven $\mathrm{Mee}^{2}$ \\ Sarah T. Pribble ${ }^{1}$ \\ David F. Siems ${ }^{3}$
}

Open-File Report 89-562

This report is preliminary and has not been reviewed for conformity with U.S. Geological Survey editorial standards or with the North American Stratigraphic Code. Any use of trade, product, or firm names is for descriptive purposes only and does not imply endorsement by the U.S. Government.

${ }^{1}$ Menlo Park, California $\quad$ 2Reston, Virginia $\quad{ }^{3}$ Lakewood, Colorado 
Introduction

This report contains chemical analyses of rocks from lava flows, cinder cones, and shallow intrusions associated with monogenetic and shield volcanoes near Crater Lake, Oregon. All analyzed samples of this type collected by C.R. Bacon and assistants (W.A. Bartling [1979, 1980], S.W. Novak [1981], M.H. Moench [1982], C.A. Michelsen [1983], M.M. Hirschman [1984, 1985], M.H. Price [1986, 1987], A.E. Gartner [1986, 1987], and M. Hanning [1988]) are included for completeness, although analyses of samples collected from 1979-1985 have been published previously (Bruggman, and others, 1987). This report is intended to supplement a paper by Bacon (in press) on the petrology and geochemistry of these rocks.

\section{Analytical methods}

Approximately 120 samples have been analyzed for major and trace elements. Chemical analyses are given in Table 1; sample number prefixes give the year of collection. Sample localities and phenocryst contents are presented in Table 2; phenocryst contents are estimates based on examination of thin sections. Samples were ground in an alumina shatterbox, except some powders for major element analyses that were made in a tungsten-carbide shatterbox. All chemical analyses were made in USGS laboratories.

Major elements were determined by wavelength-dispersive X-ray fluorescence spectrometry (WDXRF) in Lakewood, Colorado (Taggart and others, 1987). $\mathrm{Na}_{2} \mathrm{O}$ and $\mathrm{K}_{2} \mathrm{O}$ were measured by flame photometry on over half of the samples (Jackson and others, 1987). $\mathrm{Ni}, \mathrm{Rb}, \mathrm{Sr}, \mathrm{Y}, \mathrm{Zr}$, and $\mathrm{Ba}$ were determined by energy-dispersive X-ray fluorescence spectrometry (Johnson and King, 1987). $\mathrm{Nb}$ analyses for most samples collected from 1979-1986 were made by spectrophotometry; 1987-1988 samples were analyzed by inductively coupled-plasma atomic emission spectrometry (ICP) following chemical separation. Emission spectrographic methods were used to determine $\mathrm{Be}, \mathrm{Cu}, \mathrm{Ga}, \mathrm{V}$, and a few low-level $\mathrm{Rb}$ concentrations (Golightly and others, 1987). Instrumental neutron activation analysis (INAA) was used to obtain concentrations of $\mathrm{Co}, \mathrm{Cr}, \mathrm{Cs}, \mathrm{Hf}, \mathrm{Sb}, \mathrm{Ta}$, Th, U, Zn, Sc, La, Ce, Nd, Sm, Eu, Tb, Yb, and Lu (Baedecker and McKown, 1987).

\section{Precision and Accuracy}

Precision was measured by repeated analysis of internal standards. Means and standard deviations for repeated determinations of concentrations in representative rock standards are given in Table 2 of Bruggman and others (1987). Precision of wet chemical and ICP determinations were not monitored by this method; for these techniques data are reported to a number of significant figures justified by experience with the particular technique employed and concentration range encountered.

Accuracy was addressed by Bruggman and others (1987). Trace element concentrations determined for USGS standards generally agree with accepted values to within limits of measured precision. Major element data compare favorably with analyses by other laboratories of the same rock powders or of samples collected from the same outcrops, suggesting that the analyses are probably accurate to within the measured precision. $\mathrm{Na}_{2} \mathrm{O}$ is an exception in that WDXRF values prior to 1987 were consistently about $5 \%$ low relative to flame photometric values. To correct for this bias we have used flame photometric $\mathrm{Na}_{2} \mathrm{O}$ and $\mathrm{K}_{2} \mathrm{O}$ values where available and have multiplied remaining (WDXRF) $\mathrm{Na}_{2} \mathrm{O}$ values obtained before 1987 by 1.05 .

The data are available on floppy disk in ASCII, Lotus or Excel formats. Contact P. Bruggman (USGS, 345 Middlefield Rd. MS-910, Menlo Park, CA 94025 (415) 329-5234) to obtain a copy. 


\section{$\underline{\text { References }}$}

Bacon, C.R., in press, Calc-alkaline, shoshonitic, and primitive tholeiitic lavas from monogenetic volcanoes near Crater Lake, Oregon: Journal of Petrology, in press.

Baedecker, P.A., McKown, D.M., 1987, Instrumental neutron activation analysis of geochemical samples, in Baedecker, P.A., ed., Methods for Geochemical Analysis, U.S. Geological Survey Bulletin 1770, p. H1-H14.

Bruggman, P.E., Bacon, C.R., Aruscavage, P.J., Lerner, R.W., Schwarz, L.J., Stewart, K.C., 1987, Chemical Analyses of Rocks and Glass Separates from Crater Lake National Park and Vicinity, Oregon, U.S. Geological Survey Open-file Report 87-57, 36 pages.

Golightly, D.W., Dorrzapf, A. F., Jr., Mays, R.E., Fries, T.F., and Conklin, N.M., 1987, Analysis of Geologic Materials by Direct-Current Arc Emission Spectrography and Spectrometry, in Baedecker, P.A., ed., Methods for Geochemical Analysis, U.S. Geological Survey Bulletin 1770, p. A1-A13.

Jackson, L.L., Brown, F.W., and Neil, S.T., 1987, Major and minor elements requiring individual determination, classical whole rock analysis, and rapid rock analysis, in Baedecker, P.A., ed., Methods for Geochemical Analysis, U.S. Geological Survey Bulletin 1770, p. G1G23.

Johnson, R.G., King, B.-S.L., 1987, Encrgy-dispersive X-ray fluorescence spectrometry, in Baedecker, P.A., ed., Methods for Geochemical Analysis, U.S. Geological Survey Bulletin 1770, p. F1-F5.

Taggart, J.E., Jr., Lindsay, J.R., Scott, B.A., Vivit, D.V., Bartel, A.J., and Stewart, K.C., 1987, Analysis of geologic materials by wavelength-dispersive X-ray fluorescence spectrometry, in Baedecker, P.A., ed., Methods for Geochemical Analysis, U.S. Geological Survey Bulletin 1770, p. E1-E19. 


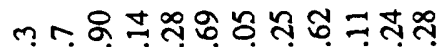

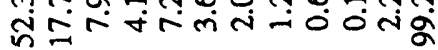

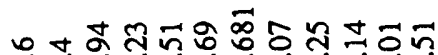

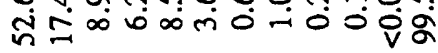

ก

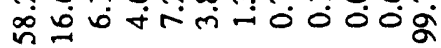

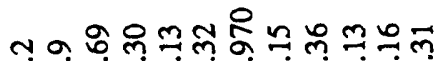
जิ

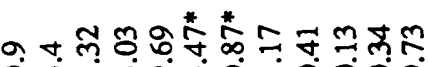

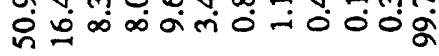

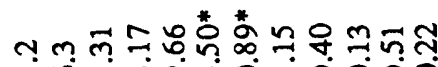

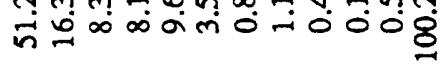

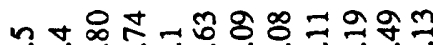

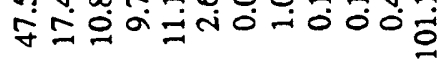

บุ

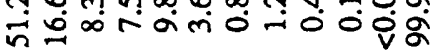

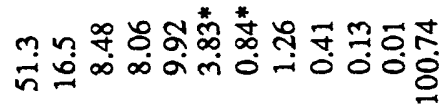

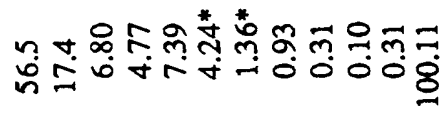

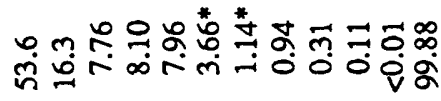

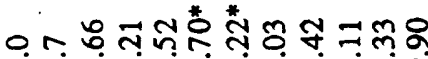

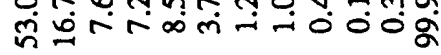

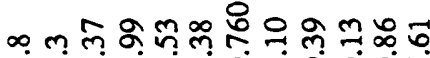

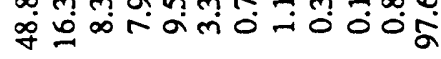

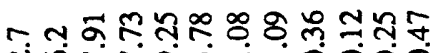
तुं

z

产

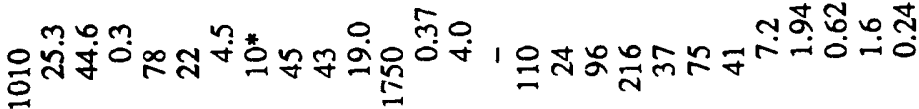

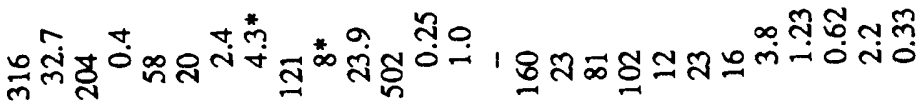

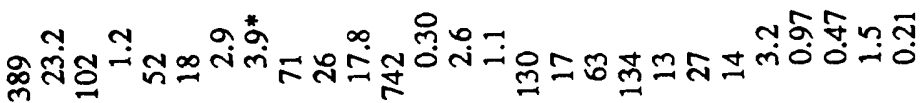

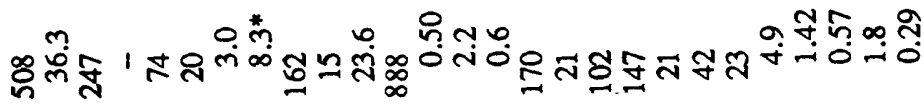

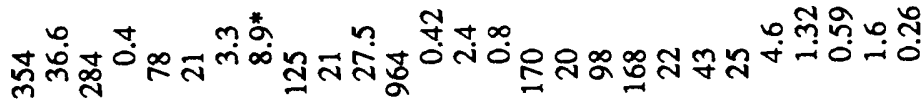

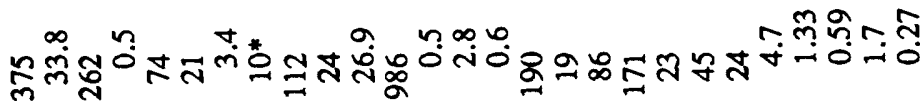

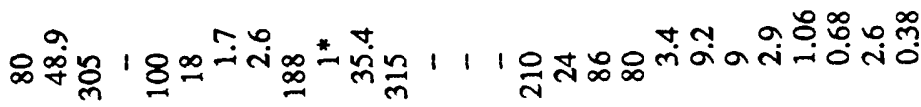

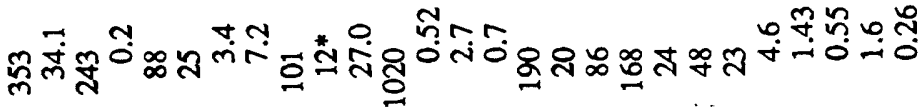
ळ

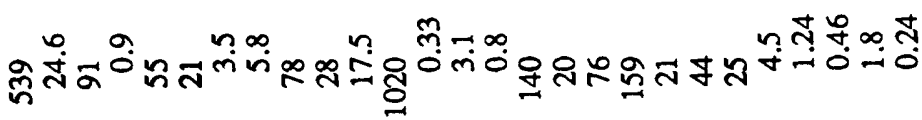

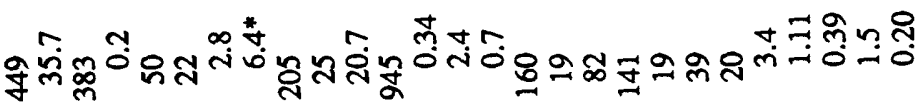

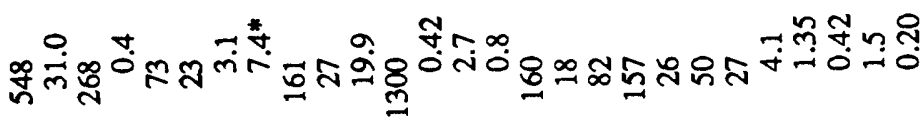

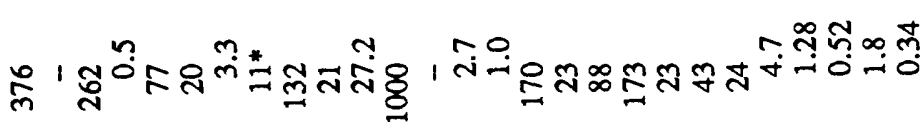

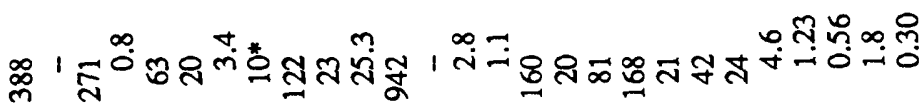

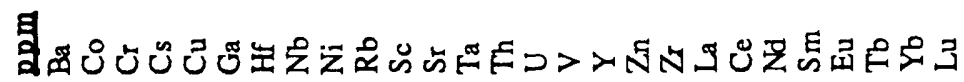

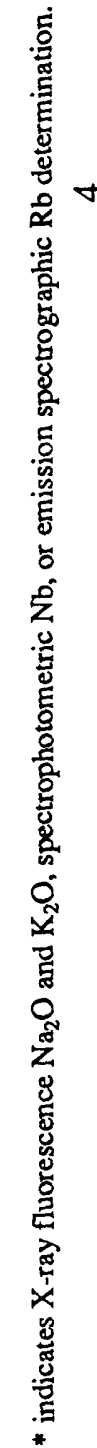




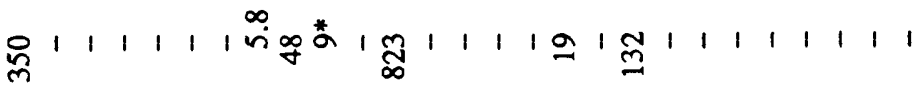

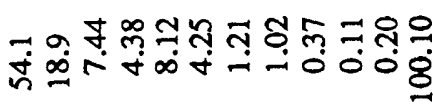

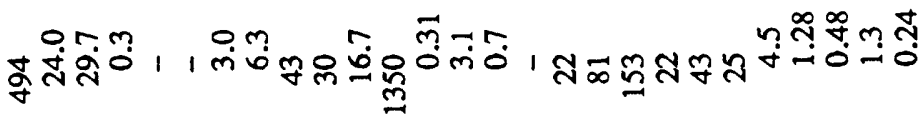

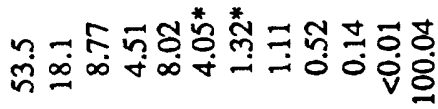

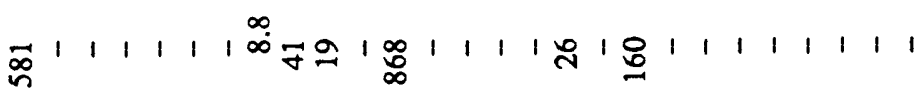

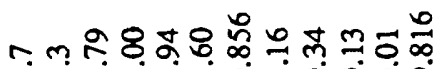
जิ

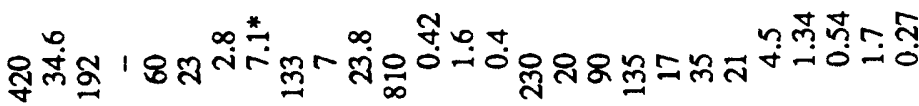

ซ.

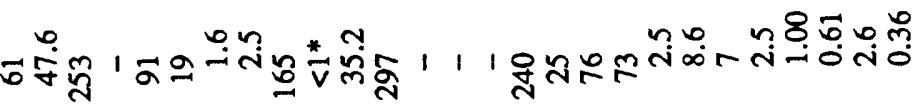

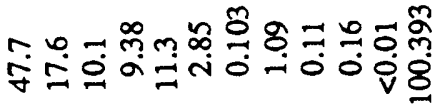

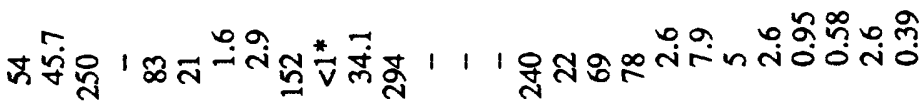

จทกรัง लिं

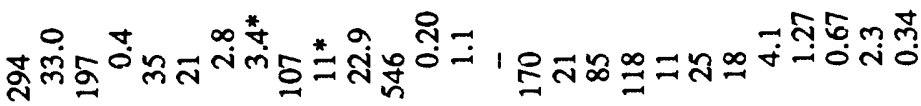

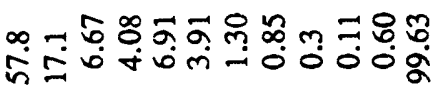

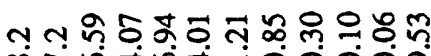

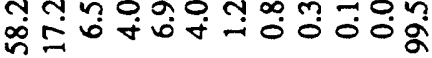

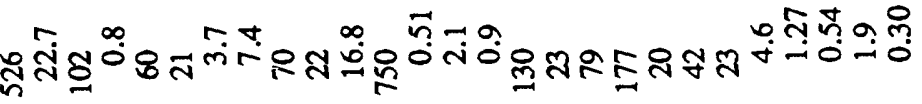

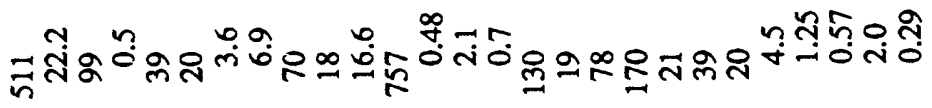

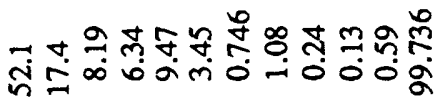

号的芦

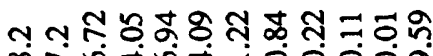

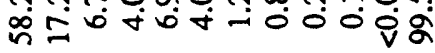

ํํำ

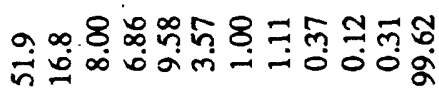

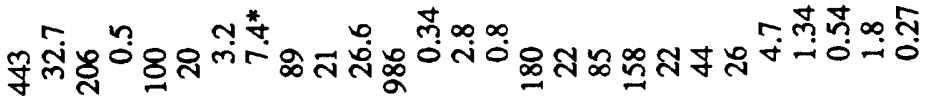

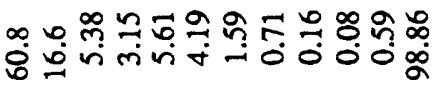

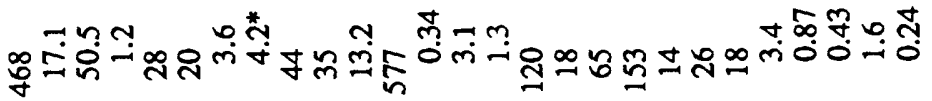

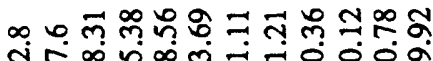

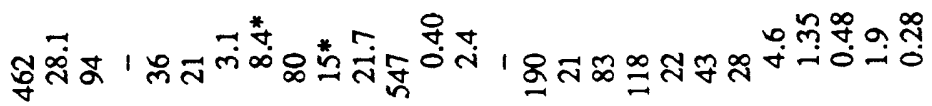




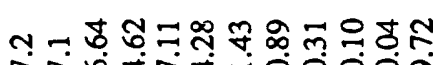

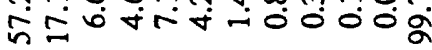

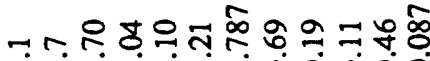

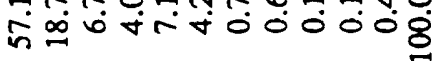

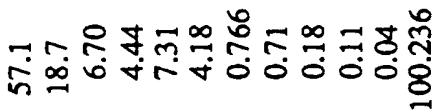

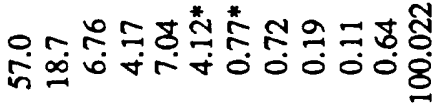

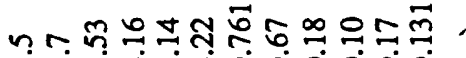

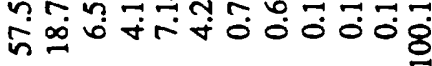

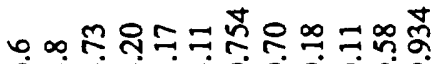

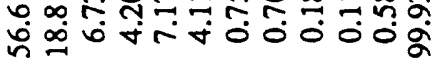

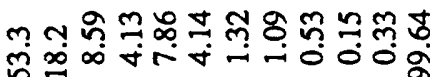

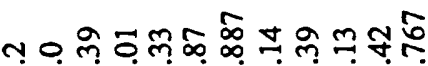

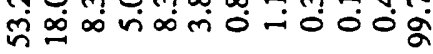

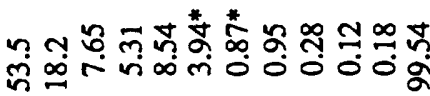

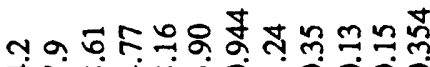

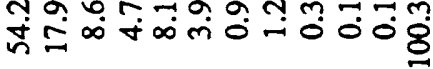

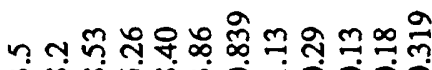

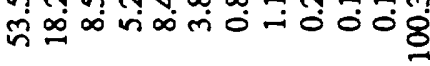

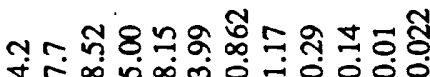

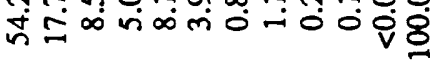

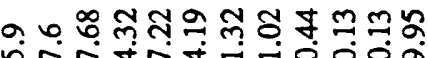

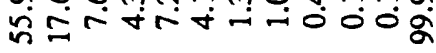

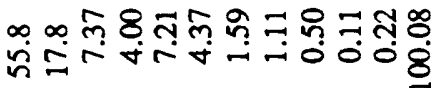

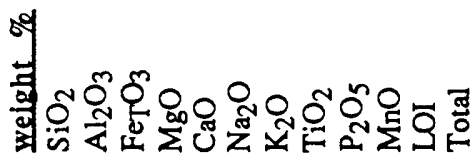

Z

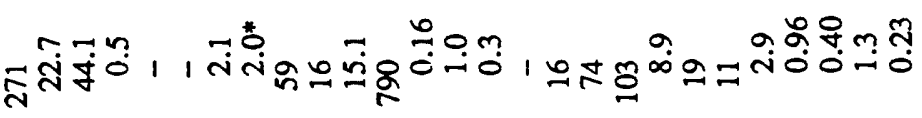

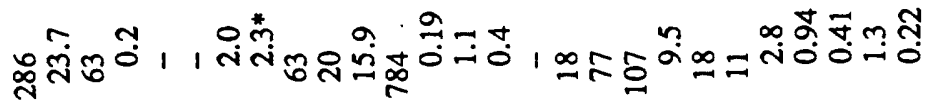
幽1111111

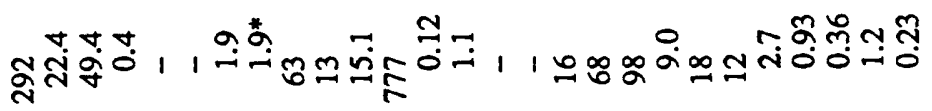

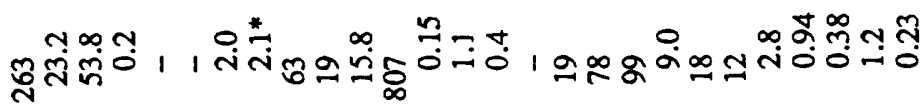

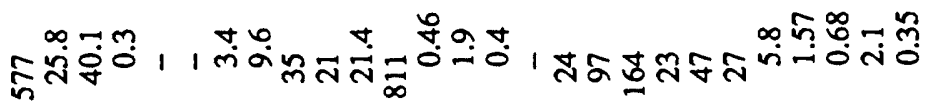

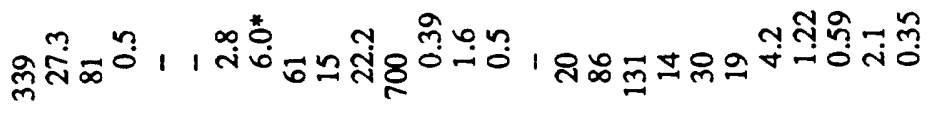

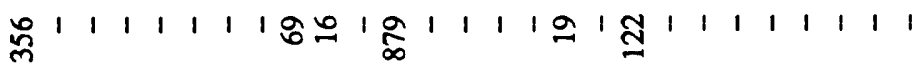

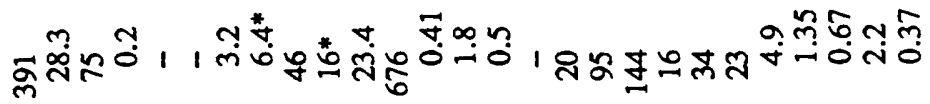

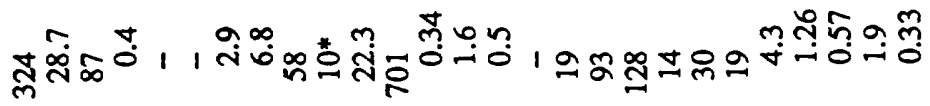

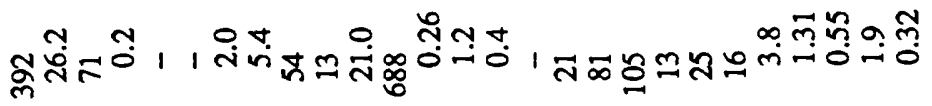

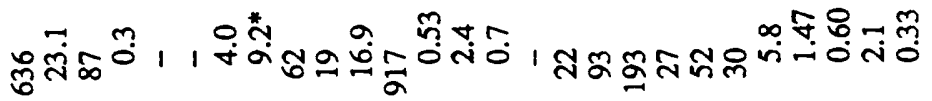

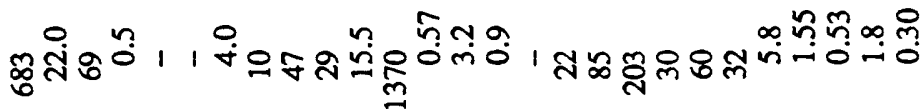




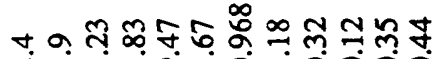
กี่

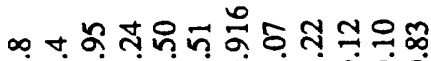

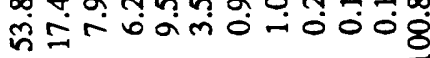

ที่ง

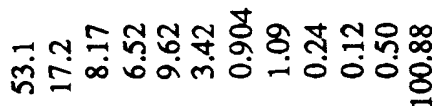

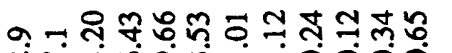

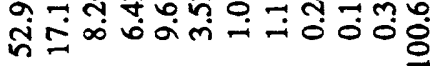

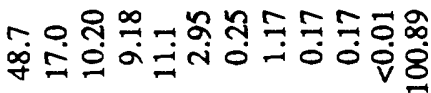

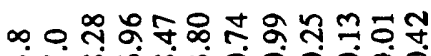

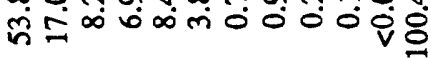

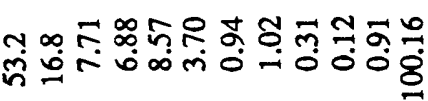

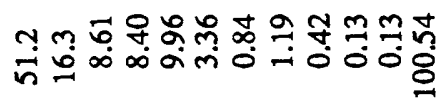

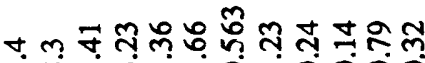

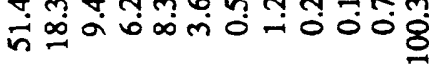

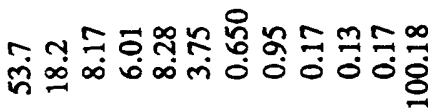

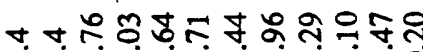

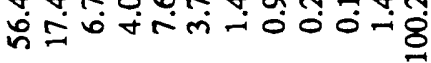

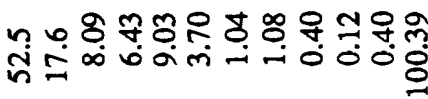

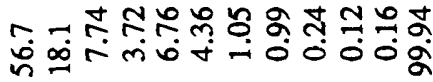

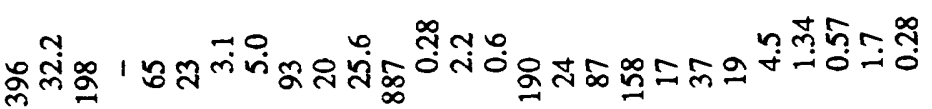

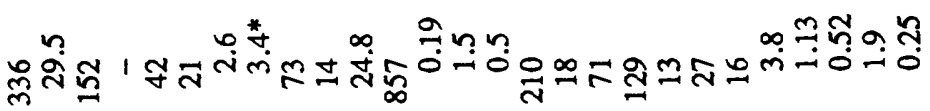

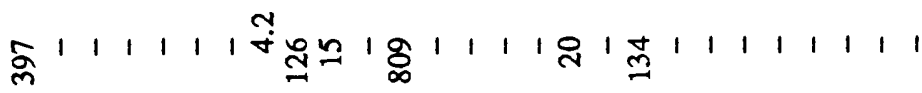

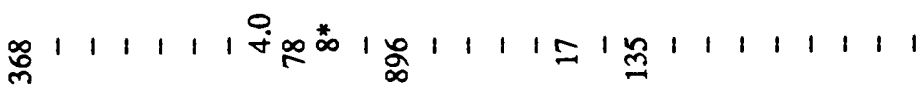

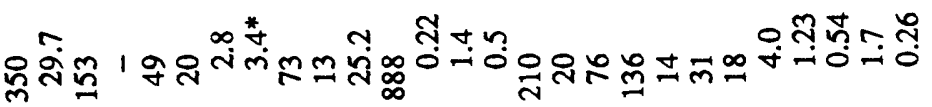

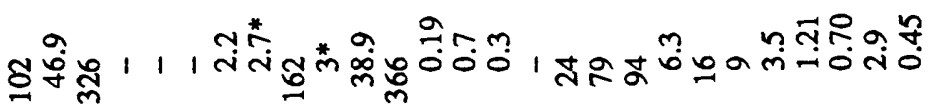
芦芦资1 1 ๙

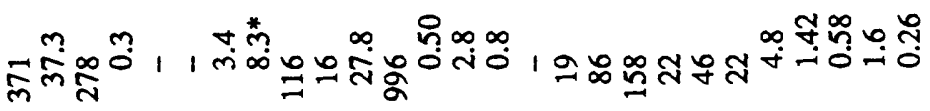

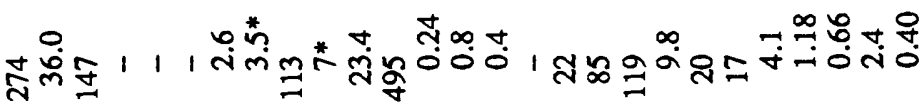

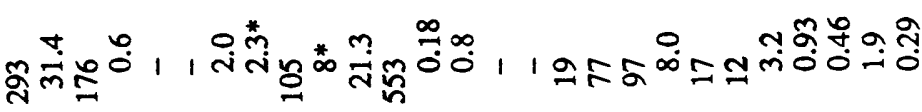

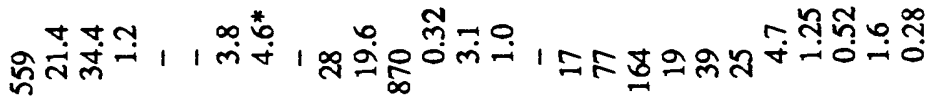

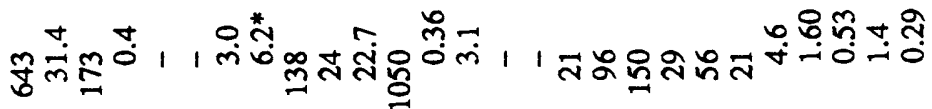

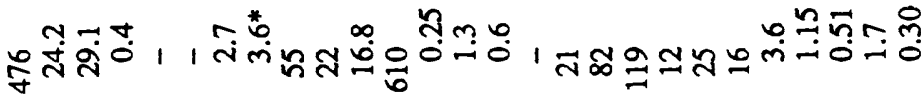




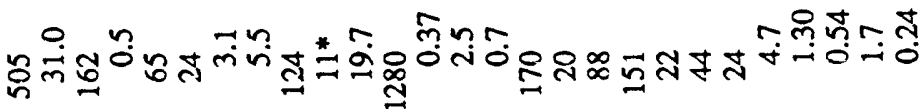

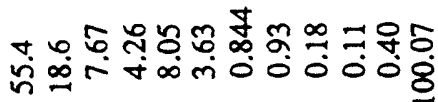

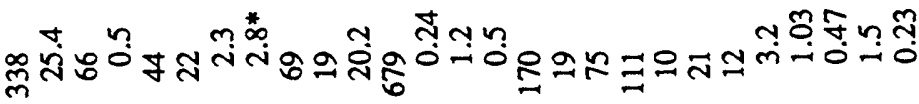

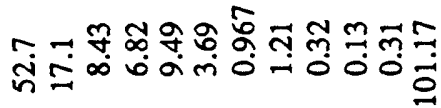

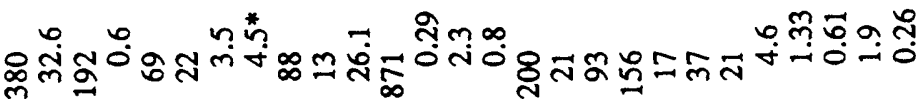

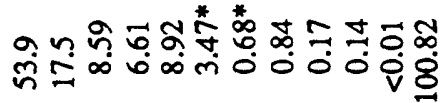

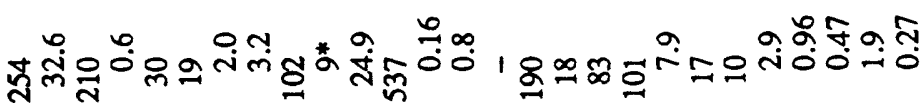

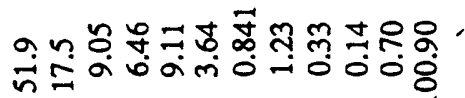

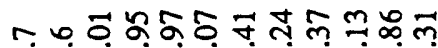

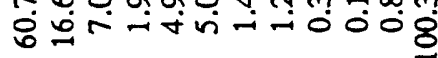

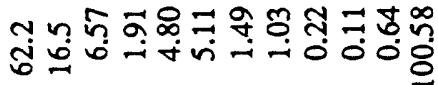

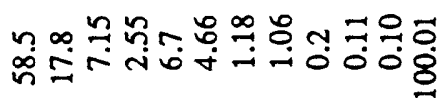

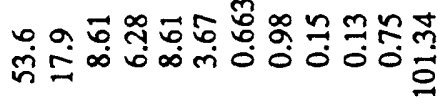

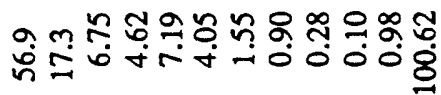

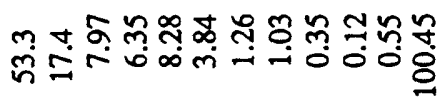

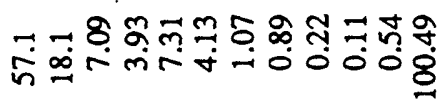

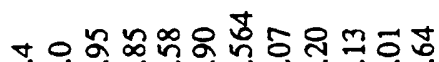

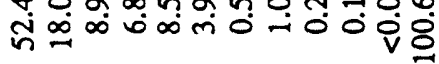

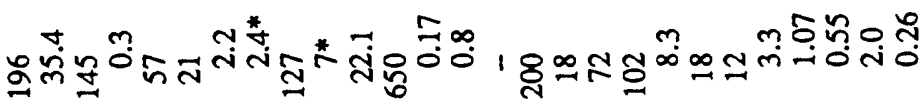
ам niñ

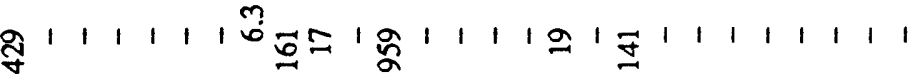

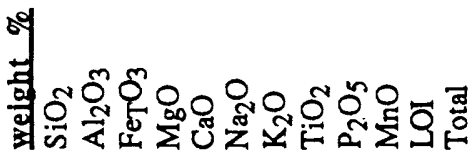




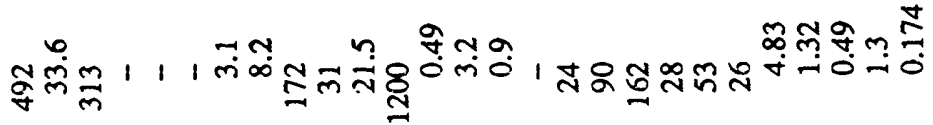

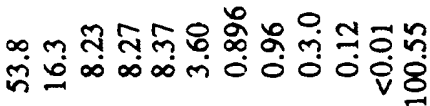

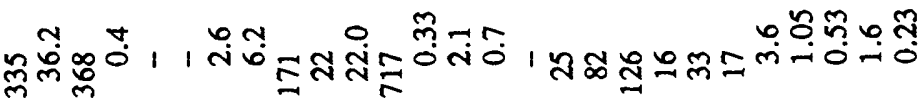

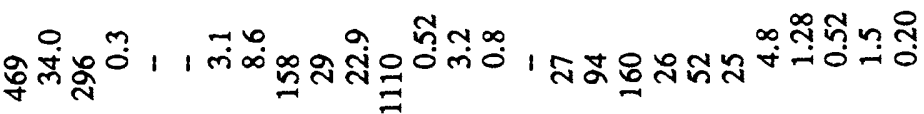

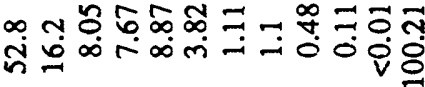

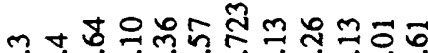

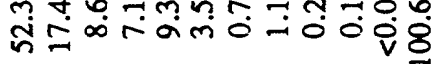

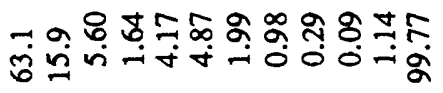

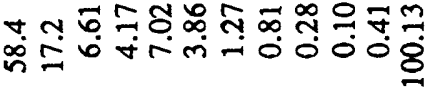

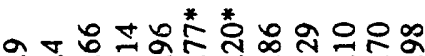

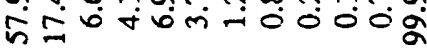

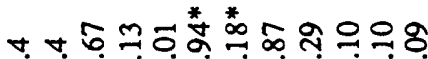

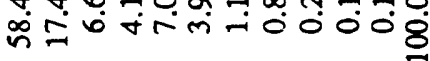

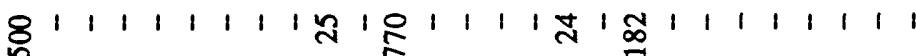

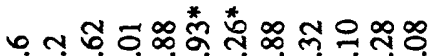

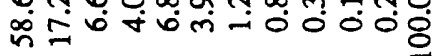

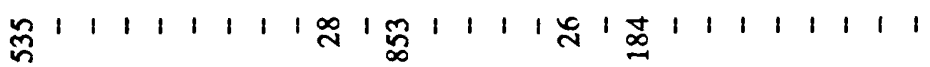
n

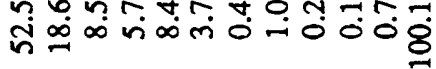

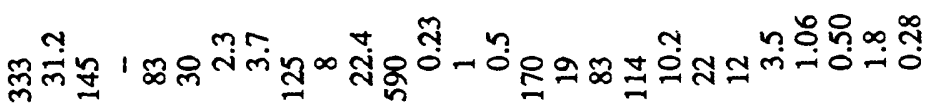

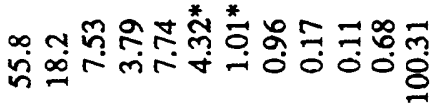

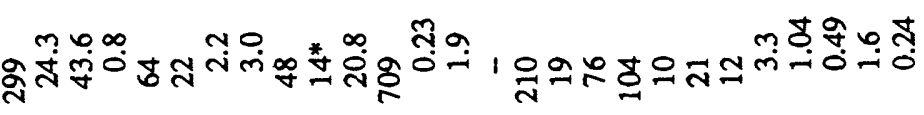

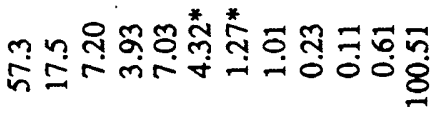

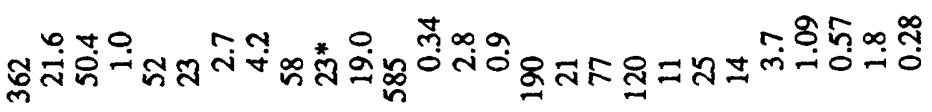

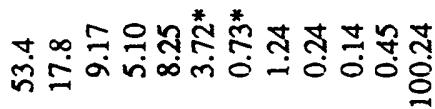

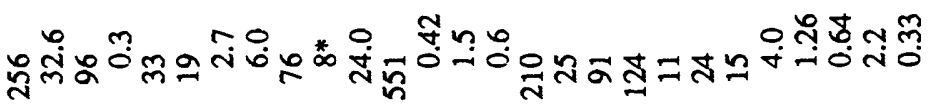

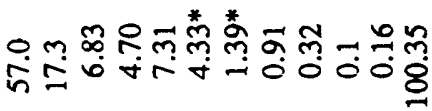

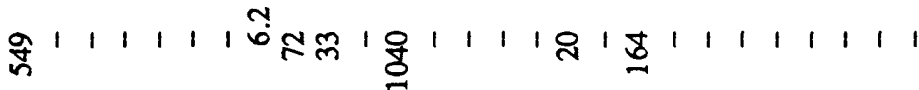

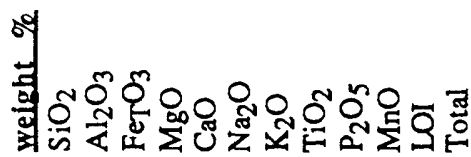




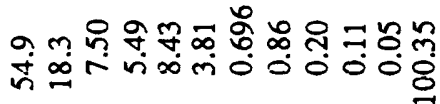

아 Ұ ๆ

जिळ

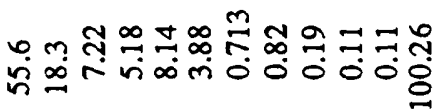

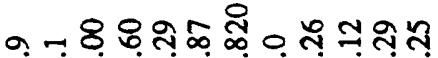

กี่

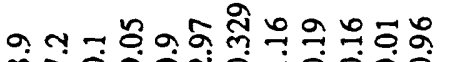

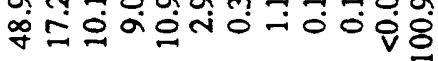

舟

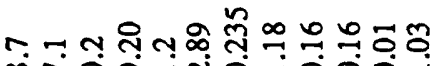

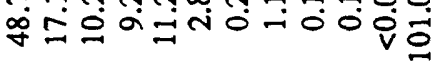

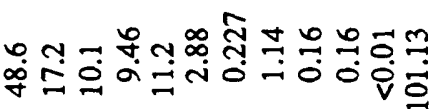

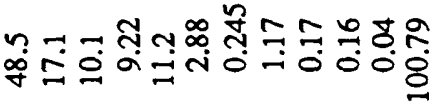

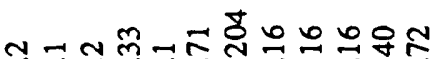

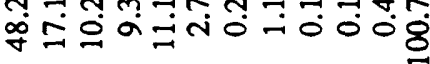

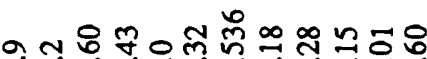

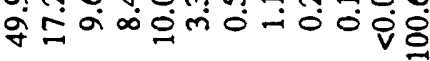

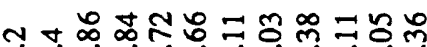

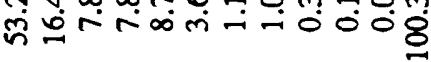

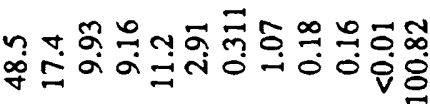

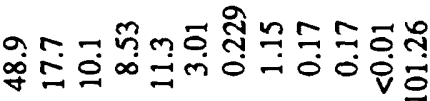

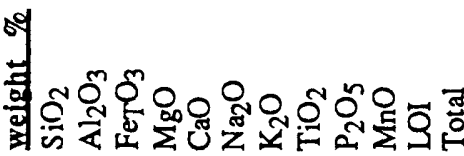

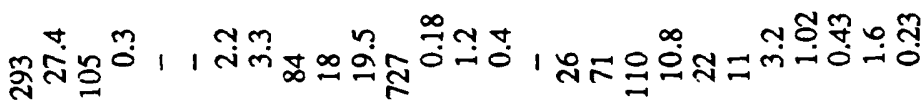

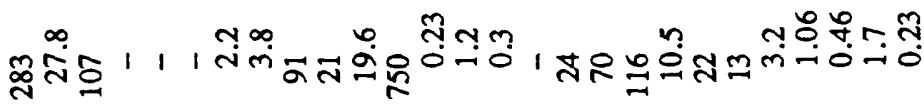

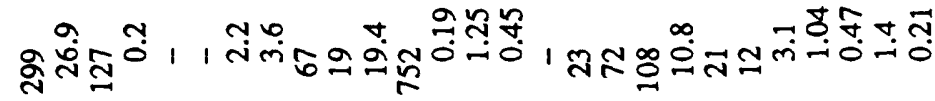

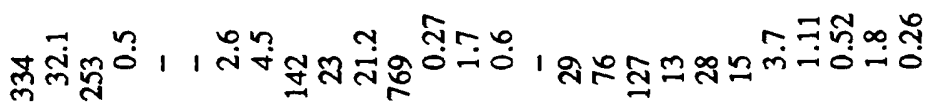

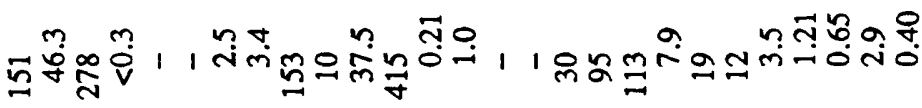

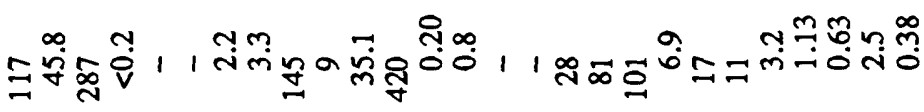

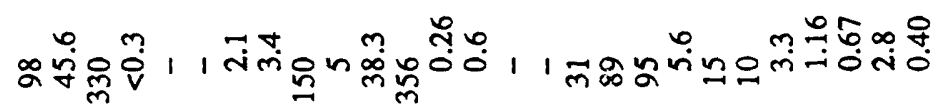

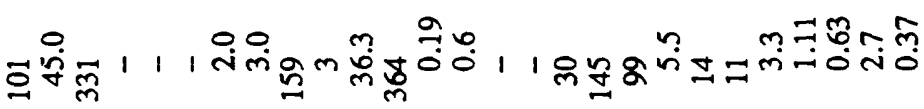

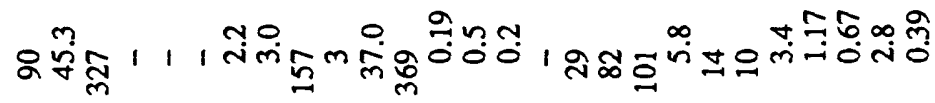

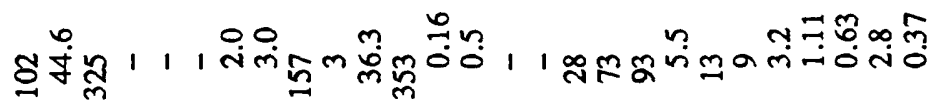

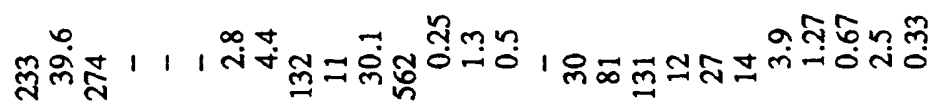

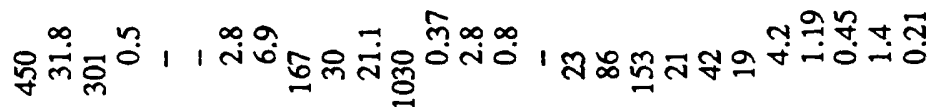

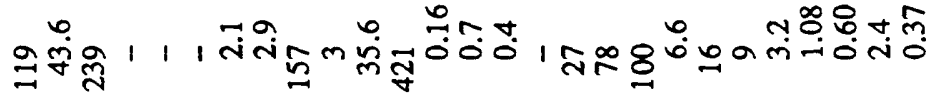

品 11 1 


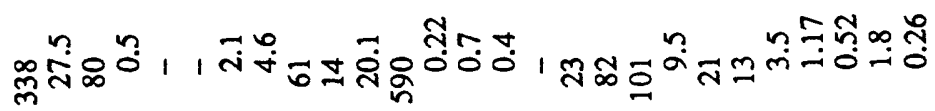

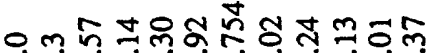

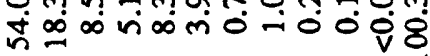

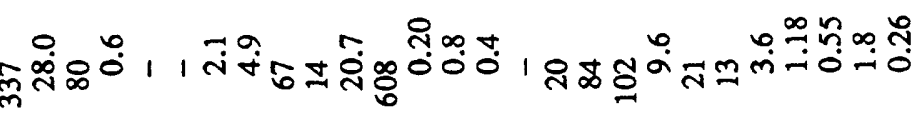

ทำณำำสำㅇํำำำ

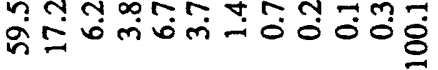

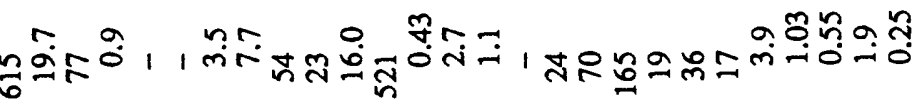

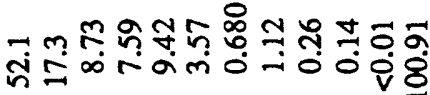

拿

ڤั

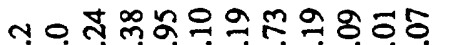

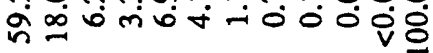

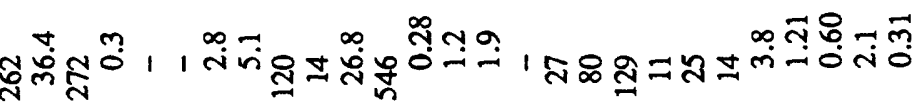

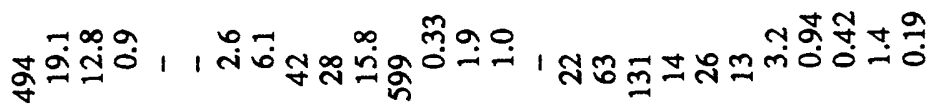

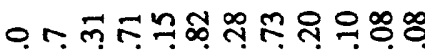

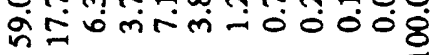

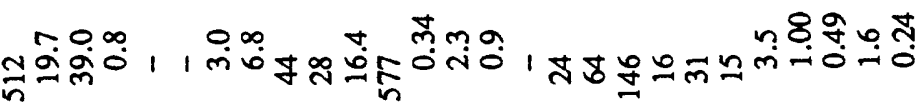

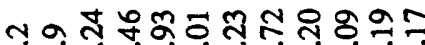

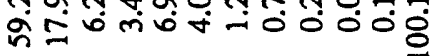

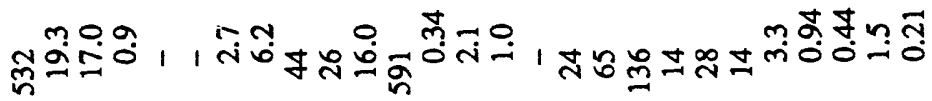

ดำ

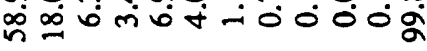

พุด ำดำํํํํํำำำ กิ่

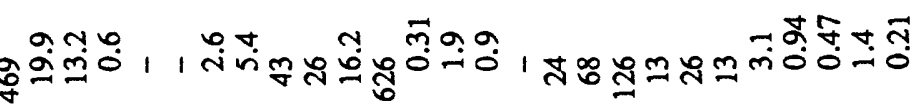

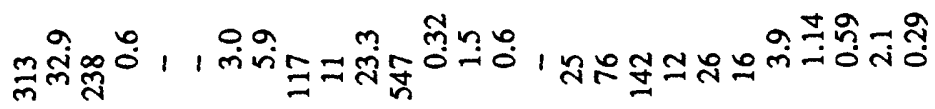

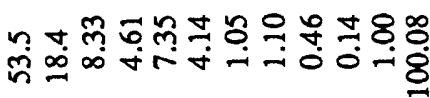

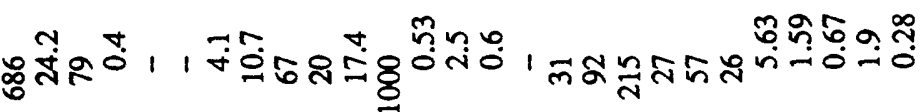

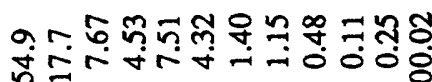

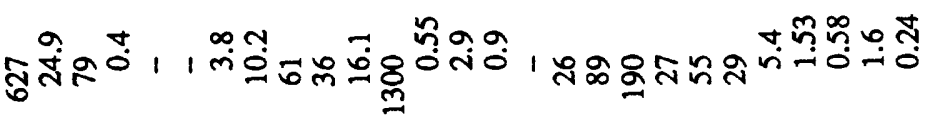

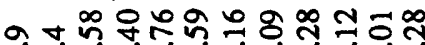

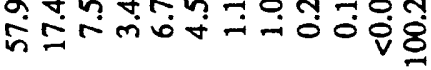

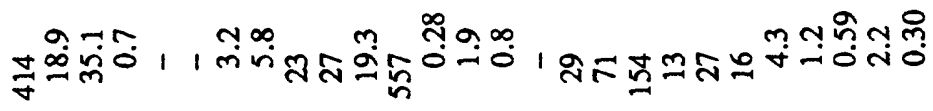

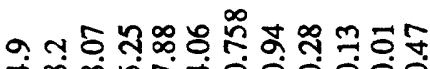

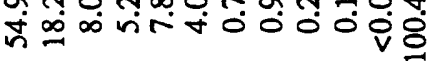

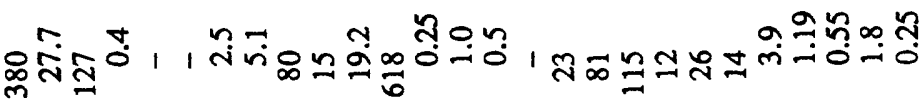

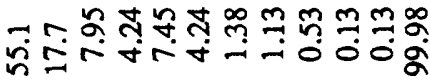

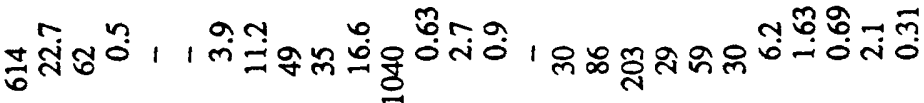

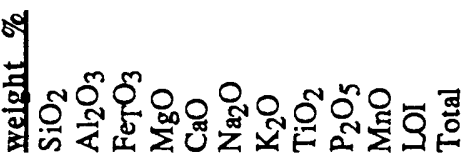




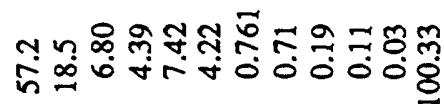

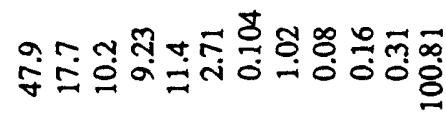

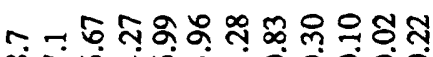

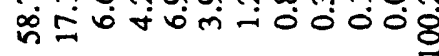

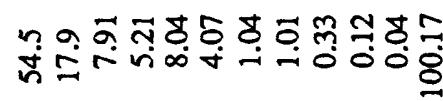

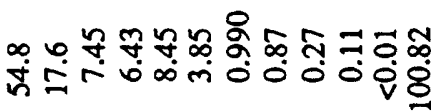

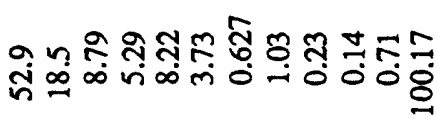

ท⿻冂从冖 ที丶

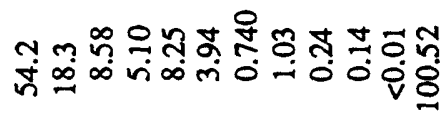
ดต

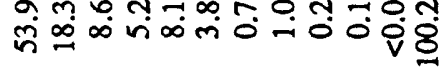
영

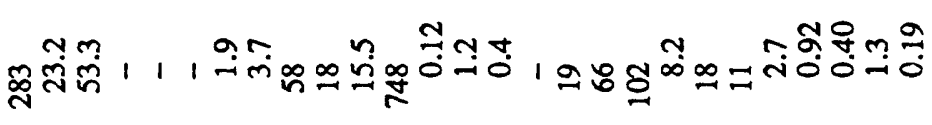
ㅁํำ

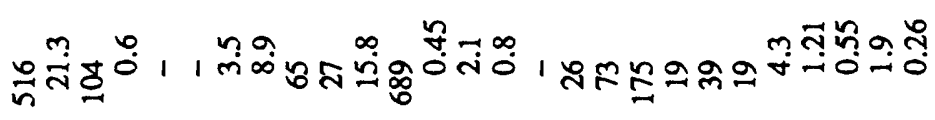

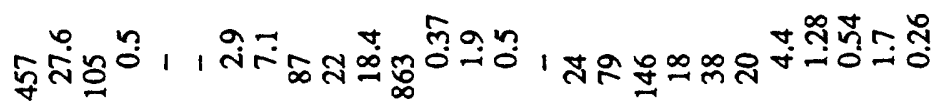

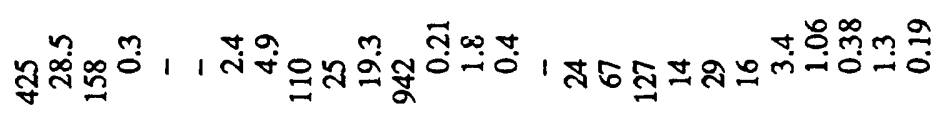

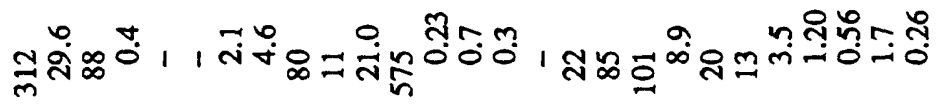
‡ู่ำ

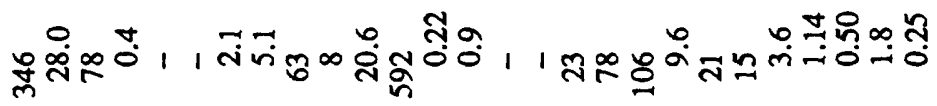

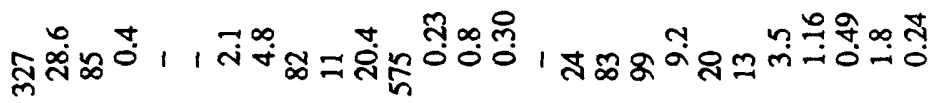

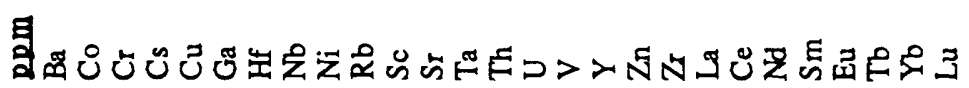




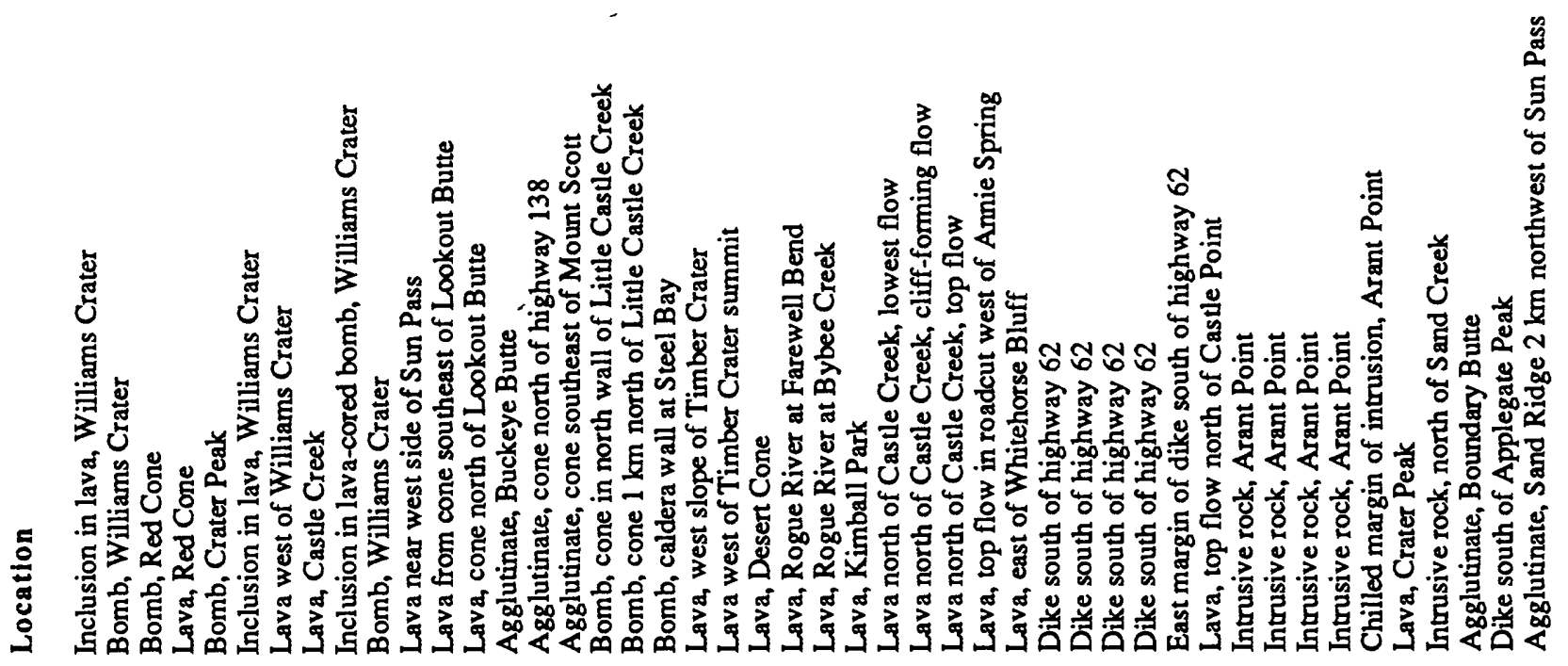

$\underset{2}{2}$

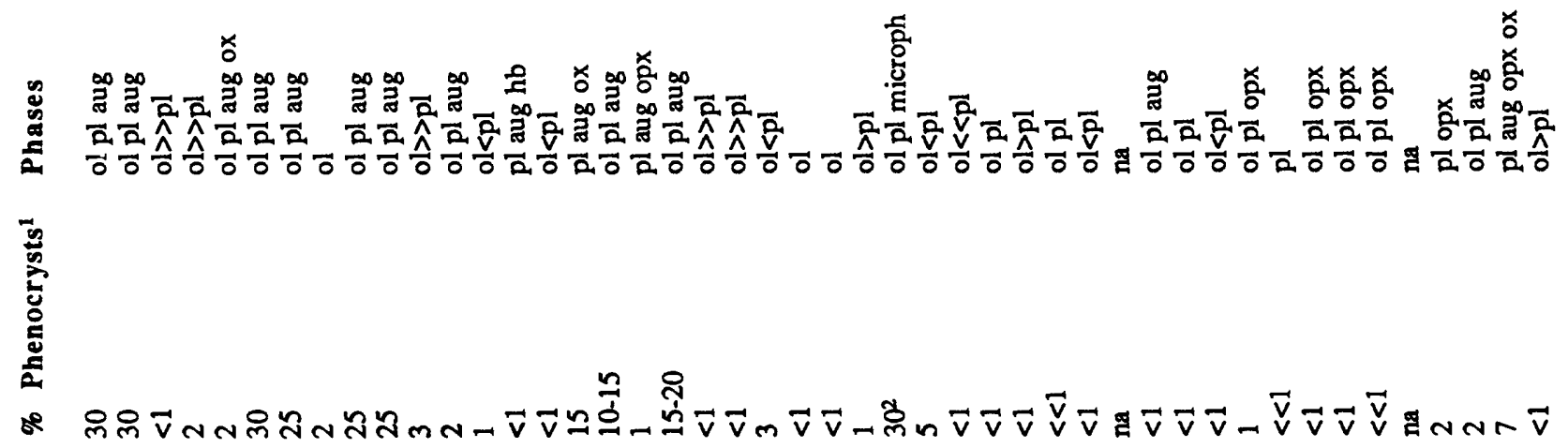

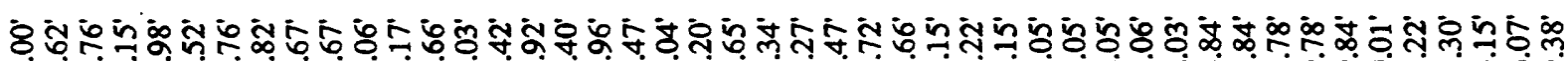

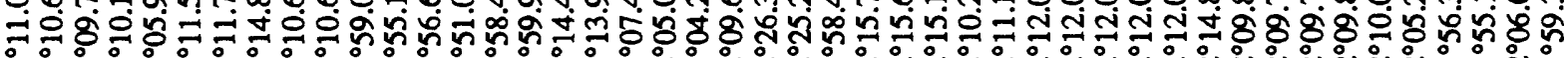
สิ่ป๋

สุล

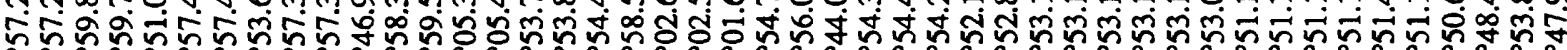

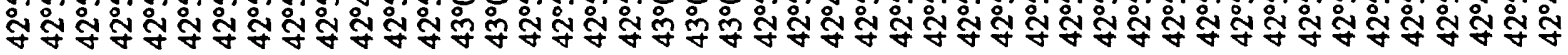

$\dot{\mathrm{z}}$

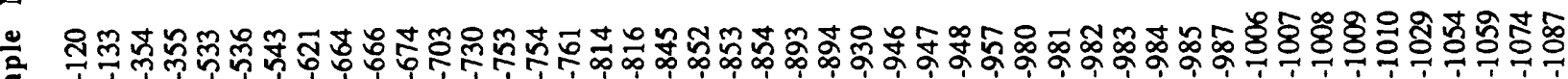

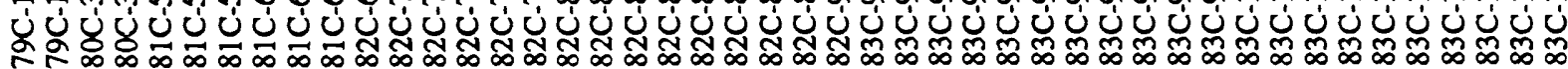




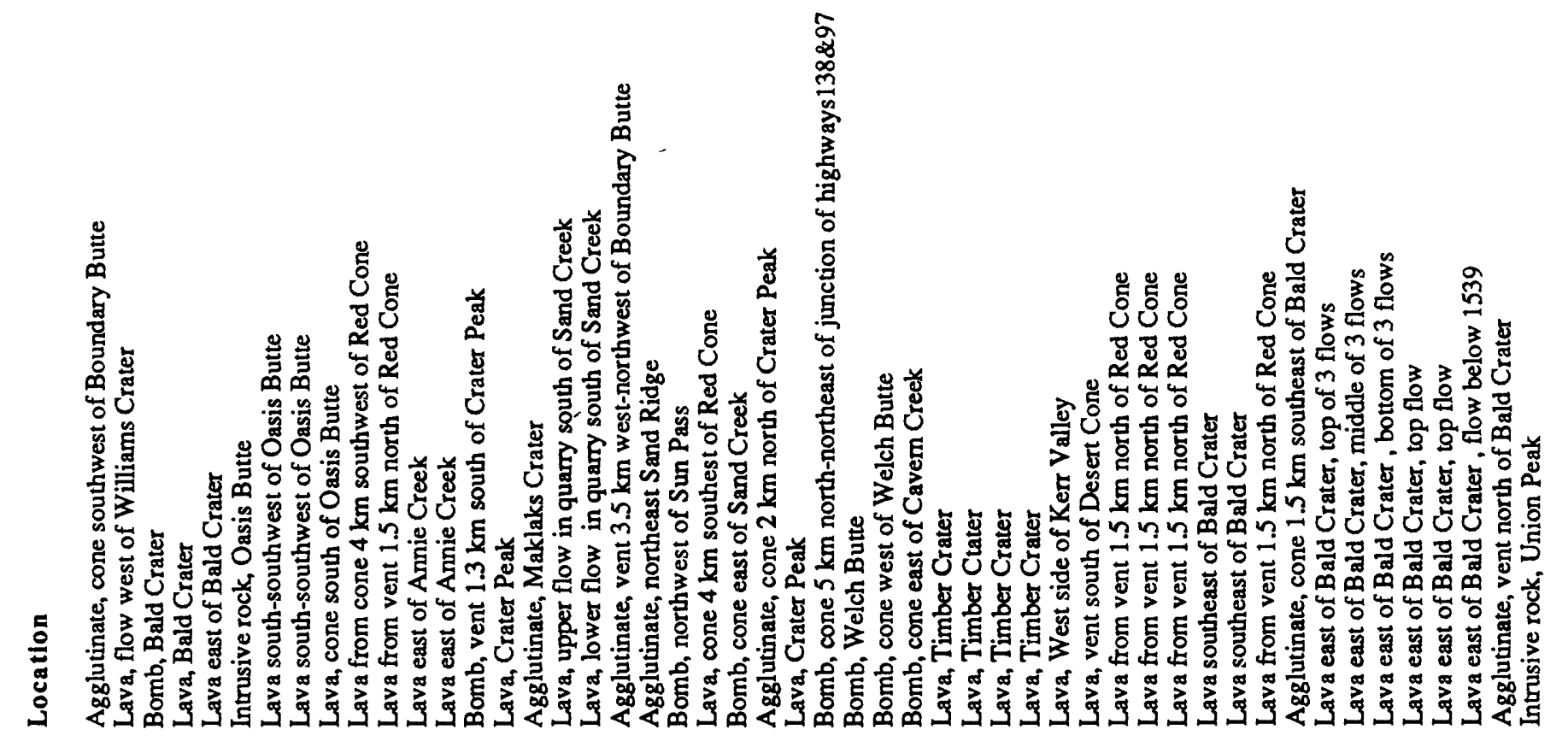

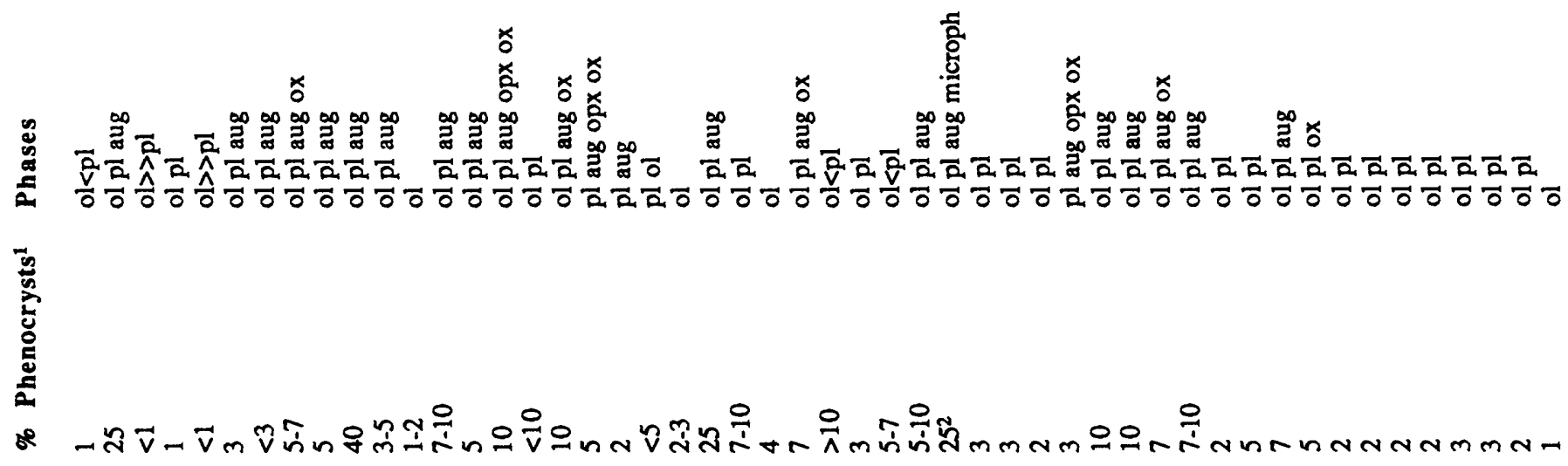

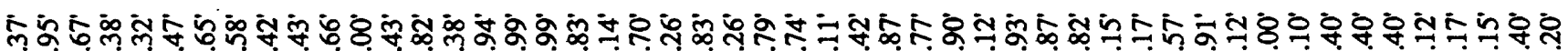

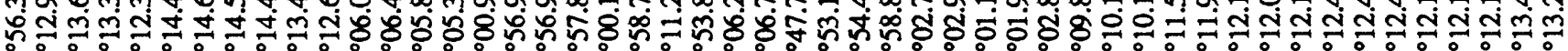
जิ

ह

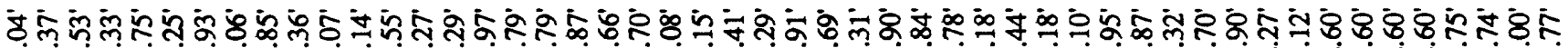

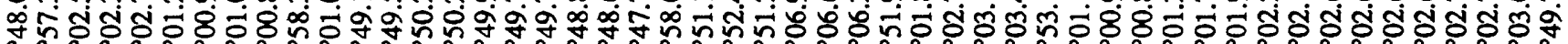

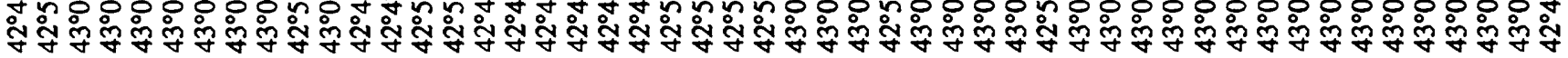

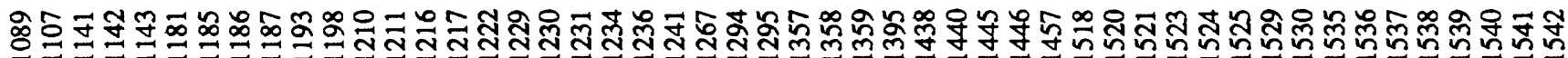

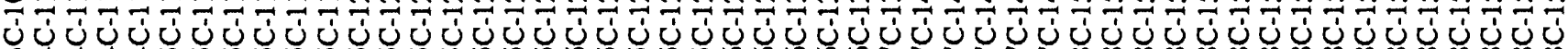

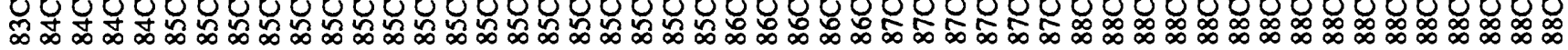



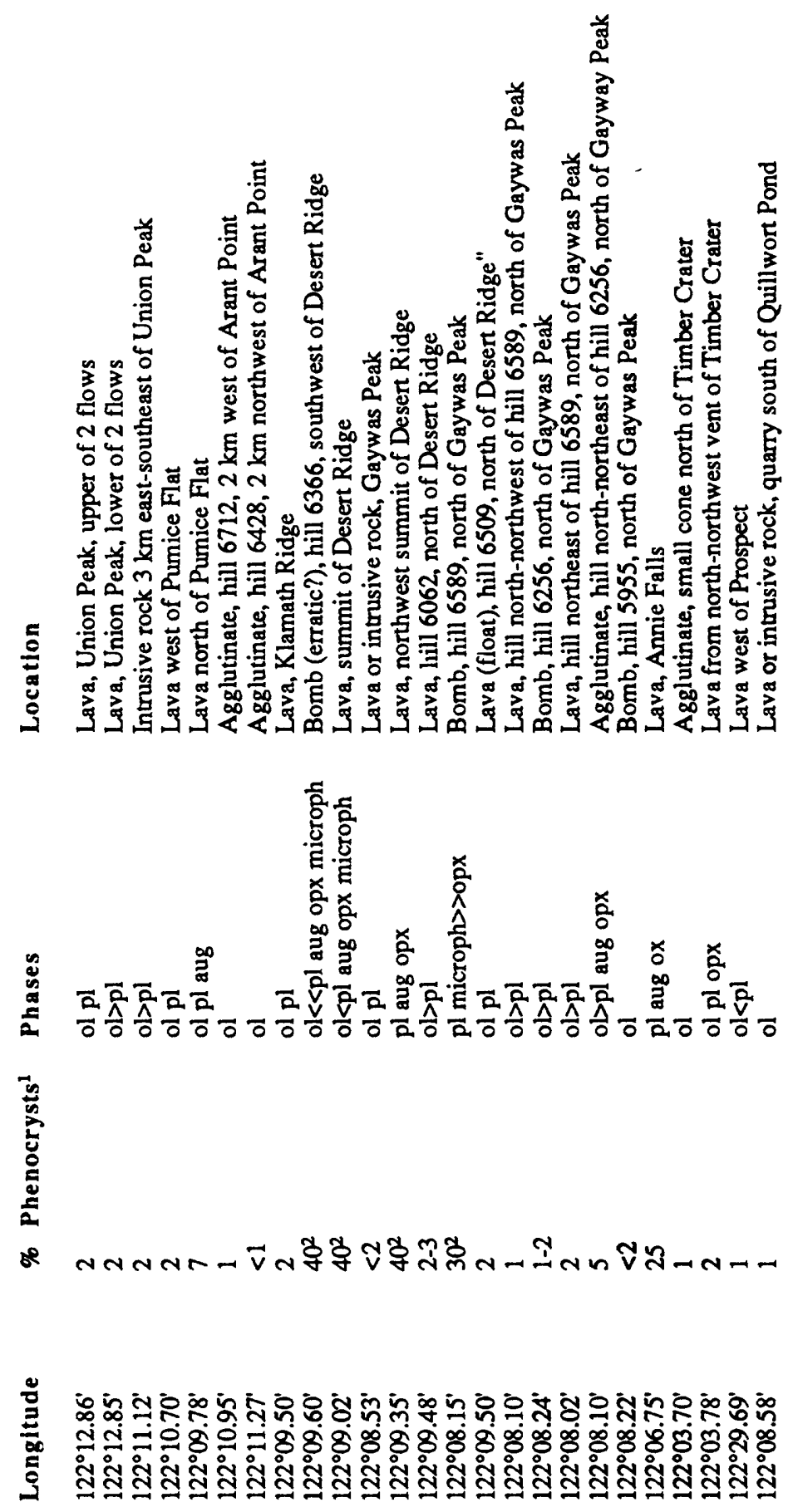

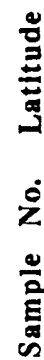

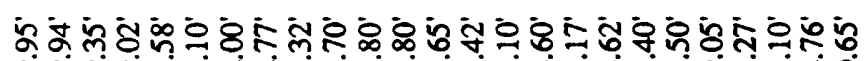

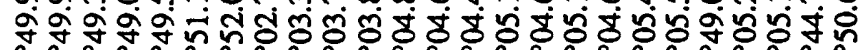

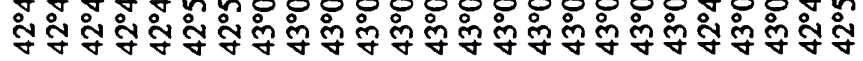

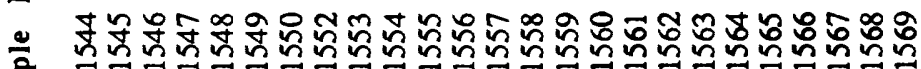

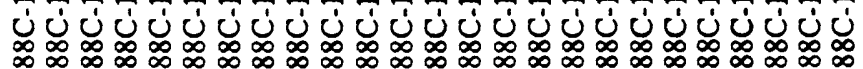

